\title{
Erythematous groin plaque in a patient with multiple myeloma
}

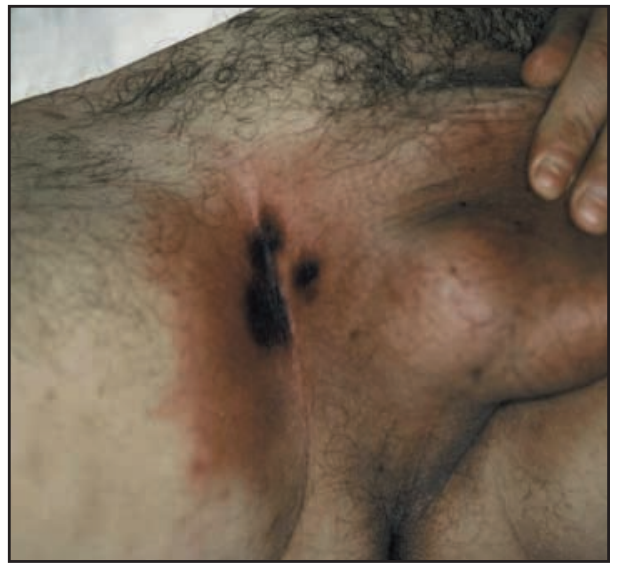

A 61-year-old white man

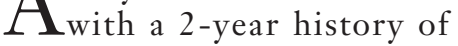
multiple myeloma presented with a painful lesion in his right groin, without a precipitating history of trauma. He had previously received systemic chemotherapy and, 8 days before presentation, intravenous cyclophosphamide.

Physical examination revealed a tender erythematous plaque with a central area of purpura localized to the right groin (Fig. 1). The patient was febrile $\left(39.5^{\circ} \mathrm{C}\right)$ and neutropenic (absolute neutrophil count $0.7 \times 10^{9} / \mathrm{L}$ ), and intravenous antibiotics (timentin and gentamycin) were started immediately. By the following morning, the lesion had progressed extensively (Fig. 2). Histopathologic examination of the skin biopsy sample revealed acute vasculitis with gram-negative rods (Fig. 3), and blood cultures subsequently grew Pseudomonas aeruginosa. The patient underwent extensive debridement of the groin, with good postoperative recovery.

The clinicopathologic features of the case were consistent with the diagnosis of ecthyma gangrenosum (EG), a rare condition and a characteristic cutaneous finding in patients with lifethreatening Pseudomonas aeruginosa bacteremia. EG typically begins as a painless erythematous macule, which develops into a hemorrhagic gray-black bulla. The bulla subsequently ruptures, leaving a central area of necrosis and gangrenous ulceration. Progression is rapid, usually evolving in 12-24 hours. ${ }^{1}$ EG lesions can occur anywhere but are most commonly found in moist sites, particularly in the groin, axillae and perianal areas.

$\mathrm{EG}$ is seen almost exclusively in immunocompromised patients, particularly those with underlying hematologic malignancy, uncontrolled diabetes mellitus, chemotherapy and burns. ${ }^{2}$ The primary cause of EG is hematogenous dissemination of $P$. aeruginosa, with seeding to the skin and soft tissues. The differential diagnosis includes Fournier's gangrene, hypersensitivity vasculitis, polyarteritis nodosa, cryoglobulinemia, fixed drug eruption and pyoderma gangrenosum.

The histologic picture of EG is that of acute inflammation, with gram-negative bacilli present in the media and adventitia of the venules. The intima is characteristically spared. ${ }^{2}$ Lesional skin cultures are usually positive for Pseudomonas.

First described in 1897, EG was thought to be pathognomonic for $P$. aeruginosa but has more recently been demonstrated to occur with other or- ganisms, such as Aeromonas bydrophila, Aspergillus, Escheria coli and Candida species. ${ }^{3}$ The mortality rate of EG ranges from $30 \%-70 \%$. Multiple lesions, prolonged neutropenia and delay in antibiotic treatment herald a poor prognosis. ${ }^{2}$

Prompt recognition of EG in a patient with associated risk factors is critical. Since progression is rapid, immediate antibiotic therapy can be lifesaving. .,2 $^{1,2}$ After appropriate cultures are obtained, patients with EG are given antipseudomonal extended spectrum penicillin in combination with an aminoglycoside, and large necrotic areas are surgically debrided. ${ }^{2}$

\section{Anatoli Freiman}

Khue Nguyen

Channy Y. Muhn

Herbert Srolovitz

Robin C. Billick

Division of Dermatology

McGill University Health Centre

Montréal, Que.

\section{References}

1. Secord E, Mills C, Shah B, Tunnessen WW Jr. Ecthyma gangrenosum secondary to Pseudomonas aeruginosa. Am 7 Dis Child 1993;147:795-6.

2. Greene SL, Su WPD, Muller SA. Ecthyma gangrenosum: report of clinical, histopathologic, and bacteriologic aspects of eight cases. $7 \mathrm{Am}$ Acad Dermatol 1984;11:781-7.

3. Reich HL, Williams Fadeyi D, Naik NS, Honig PJ, Yan AC. Nonpseudomonal ecthyma gangrenosum. 7 Am Acad Dermatol 2004;50[5 Suppl]:S114-7. 